\title{
Análise do conhecimento e prática da população de Vitória da Conquista-Bahia
}

\section{sobre descarte de medicamentos}

\author{
Analysis of drug disposal knowledge and practices of the population of Vitória da Conquista, Bahia \\ Análisis del conocimiento y la práctica de la población de Vitória da Conquista-Bahia sobre la \\ eliminación de medicamentos
}

Recebido: 21/11/2021 | Revisado: 01/12/2021 | Aceito: 02/12/2021 | Publicado: 13/12/2021

\author{
Ana Maria Reis Souza \\ ORCID: https://orcid.org/0000-0002-1249-8083 \\ Faculdade Independente do Nordeste, Brasi \\ E-mail: reisana281@hotmail.com \\ Graziely Oliveira Bonfim \\ ORCID: https://orcid.org/0000-0002-1150-4584 \\ Faculdade Independente do Nordeste, Brasil \\ E-mail: grazybonfim@hotmail.com \\ Larissa Morgan Andrade Lemos \\ ORCID: https://orcid.org/ 0000-0001-6148-3825 \\ Faculdade Independente do Nordeste, Brasil \\ E-mail: larissamorgan@fainor.com.br
}

\begin{abstract}
Resumo
Apesar do elevado consumo de medicamentos pela população mundial, observa-se que há difusa falta de orientação sobre o descarte adequado desses produtos. O presente estudo refere-se a um problema capaz de atingir a saúde coletiva, tendo em vista que os medicamentos são considerados como resíduos do grupo B, no qual englobam substâncias químicas que podem expor risco à saúde pública ou ao meio ambiente. Frente ao exposto, esta pesquisa apresenta como objetivos: analisar como a população de Vitória da Conquista - BA realiza o descarte de medicamentos em geral. Trata-se de uma pesquisa de natureza aplicada, descritiva e exploratória e de abordagem quantitativa. Para a coleta de dados, foi utilizado um instrumento estruturado contendo 11 questões que abordam o perfil socioeconômico demográfico dos participantes, bem como, o grau de conhecimento acerca do descarte de medicamentos vencidos/desuso. A coleta de dados foi realizada em pontos estratégicos da cidade, onde existe um alto fluxo de pessoas que residem em diversos bairros. Foram entrevistadas 207 habitantes maiores de 18 anos, de ambos os sexos, contando como critério de exclusão crianças e adolescentes, pois esses possuem pouca probabilidade de serem os responsáveis pelo descarte de medicamentos de suas residências. Para a organização e análise dos dados coletados através das abordagens em campo, foi utilizado o programa Microsoft Excel 2019. Dentre os entrevistados, foi observado uma maior predominância do sexo feminino $(52,17 \%)$, considerando que, $51,82 \%$ da população de Vitória da Conquista são do sexo feminino. Os resultados encontrados no presente estudo evidenciam que a população de Vitória da Conquista descarta os medicamentos de forma incorreta. Conclui-se que há um déficit de conhecimento acerca do descarte adequado em decorrência da falta de informação sobre o tema, carência de pontos de coleta e falta de divulgação dos problemas ambientais provocados pelo descarte.
\end{abstract}

Palavras-chave: Medicamento; Resíduo; Contaminação.

\begin{abstract}
In spite of the substantial consumption of drugs by the world's population, there is a large lack of orientation about how to adequately dispose of such products. This study refers to a problem that can affect public health, since these drugs are considered class B residues, which encompasses chemical substances that may represent a risk to public health and to the environment. Given the above, this research has the following objectives: to analyze how the population of Vitória da Conquista - BA performs the disposal of drugs in general. This is a descriptive, exploratory study of applied nature and a quantitative approach. An instrument with 11 questions was used for data collection, regarding the demographic and socioeconomic status of the participants, as well as their level of knowledge on the disposal of expired/unused drugs. The data collection was carried out at strategic points in the city, where there is a high flow of people living in different neighborhoods. 207 inhabitants over 18 years old and from both genders were interviewed, with the exclusion of children and teenagers, as these were less likely to be responsible for drug disposal in their households. For the organization and analysis of data collected through field research, the Microsoft Excel 2019 program was used. Among the interviewees, there was a predominance of female subjects $(52,17 \%)$, considering that $51,82 \%$ of the population of Vitória da Conquista is female. The results found in this study demonstrate that the
\end{abstract}


population of Vitória da Conquista disposes of drugs incorrectly. In conclusion, there is a knowledge deficit on appropriate disposal which originates from the lack of information on the topic, the shortage of take back locations and a lack of promotion of the environmental issues caused by the disposal.

Keywords: Drug; Residue; Contamination.

\section{Resumen}

Aunque existe un alto consumo de medicamentos por parte de la población mundial, se observa que hay una extensa falta de orientación sobre la manera correcta de desechar esos productos. Este estudio se refiere a un problema que es capaz de afectar la salud colectiva, teniendo en cuenta que los medicamentos forman parte de los residuos del grupo $\mathrm{B}$, en el que se incluyen sustancias químicas que pueden suponer un riesgo para la salud pública o para el medio ambiente. Dado lo anterior, esta investigación tiene los siguientes objetivos: analizar cómo la población de Vitória da Conquista - BA realiza la disposición de medicamentos en general. Se trata de una investigación aplicada, descriptiva y exploratoria con enfoque cuantitativo. Para la recogida de datos, se utilizó un instrumento estructurado que contenía 11 preguntas referentes al perfil demográfico y socioeconómico de los participantes, así como al nivel de conocimiento sobre la eliminación de medicamentos caducados/no utilizados. La recolección de datos se realizó en puntos estratégicos de la ciudad, donde existe un alto flujo de personas que viven en diferentes barrios. Se entrevistó a un total de 207 habitantes mayores de 18 años, de ambos sexos, se utilizó como criterio de exclusión a los niños y adolescentes, porque es poco probable que sean responsables por eliminar los medicamentos en sus hogares. Para la organización y análisis de los datos recolectados a través de enfoques de campo, se utilizó el programa Microsoft Excel 2019. Entre los entrevistados hubo un mayor predominio de mujeres $(52,17 \%)$, teniendo en cuenta que el $51,82 \%$ de la población de Vitória da Conquista es femenina. Los resultados encontrados en este estudio muestran que la población de Vitória da Conquista desecha los medicamentos de manera incorrecta. Se concluye que existe un déficit de conocimiento sobre la eliminación correcta de medicamentos debido a la falta de información sobre el tema, a la falta de centrales de recolección y a la falta de difusión sobre los problemas ambientales provocados por su incorrecta eliminación.

Palabras clave: Medicamentos; Residuo; Contaminación.

\section{Introdução}

O avanço da indústria farmacêutica e a expansão do acesso aos medicamentos colaboram para o crescimento do seu consumo e acúmulo nos domicílios. Dessa forma, os vários tipos de medicamentos não utilizados, a quantidade armazenada nas residências e as práticas de descarte, bem como os fatores que podem explicar o desaproveitamento, são temas de vários estudos (Cruz et al., 2017).

Estudos mostram que 64,6\% das mulheres e 45,4\% dos homens em idade adulta utilizam no mínimo um medicamento por dia e que $80 \%$ dos indivíduos que possuem doenças crônicas não transmissíveis utilizam medicamentos diariamente (Bandeira et al., 2019).

Apesar do elevado consumo diário de medicamentos pela população mundial, observa-se que há difusa falta de orientação sobre o descarte desses produtos e o trato com a demanda específica de resíduos (Ramos, 2017). Trata-se de um problema capaz de atingir a saúde coletiva, tendo em vista que os medicamentos são considerados como resíduos do grupo B, no qual englobam substâncias químicas que podem expor risco à saúde pública ou ao meio ambiente (Tonet et al., 2020).

Em relação à legislação, apenas em 2020 o Brasil publicou um decreto específico regulamentando o descarte de medicamentos vencidos ou em desuso. Esse decreto possibilita melhorias na realização desse descarte (Ferreira et al., 2020). Apesar de existem avanços na legislação, o gerenciamento dos resíduos ainda apresenta grandes deficiências nos aspectos de tratamento e disposição final, principalmente no que tange os resíduos de medicamentos que, em razão de suas características farmacológicas, podem se tornar tóxicos ao ambiente contaminando solos, águas subterrâneas, águas superficiais e lençóis freáticos, o que justifica a necessidade de mais pesquisas e estudos (Lopes et al., 2021; De Paula, 2019).

Estudo realizado por Constantino et al. (2020) apresentaram achados que demonstraram que o ato de instituir postos de coleta de medicamentos ou até mesmo projetos para recolhimento, não se mostraram capazes de mudar a percepção e os hábitos da população em sua rotina diária quanto ao descarte. A população carece de educação em saúde, no qual permita um maior conhecimento no que se refere às práticas adequadas para a conservação e descarte de medicamentos. A adoção de 
medidas cológicas favorecem a descaracterização apropriada dos medicamentos e devem ser incentivadas na população, considerando não só as comunidades de hoje, mas também as gerações futuras.

Frente ao exposto, esta pesquisa apresenta como objetivos: analisar como a população de Vitória da Conquista - BA realiza o descarte de medicamentos em geral; verificar a forma de descarte residencial de medicamentos; e, verificar as classes medicamentosas mais descartadas pela população de Vitória da Conquista - BA.

\section{Metodologia}

Trata-se de um estudo de natureza aplicada, que segundo Prodanov e Freitas (2013), objetiva gerar conhecimentos para aplicação prática, dirigidos à solução de problemas específicos, envolvendo características e interesses locais.

Do ponto de vista da abordagem, trata-se de uma pesquisa quantitativa, tendo como finalidade a precisão dos resultados, evitando as distorções da análise e garantindo maior margem de confiança. Para a coleta de dados foi utilizado um instrumento estruturado, previamente testado em um estudo piloto (Marcondes et al., 2017).

$\mathrm{O}$ presente estudo também pode ser classificado quanto aos seus objetivos, como uma pesquisa descritiva e exploratória. A junção dessas pesquisas são habitualmente realizadas pelos pesquisadores sociais que estão preocupados com a atuação prática (Prodanov e Freitas, 2013).

A pesquisa foi realizada no município de Vitória da Conquista, Estado da Bahia, distante 519 quilômetros de Salvador, capital do Estado, dispondo de área com $3.204 \mathrm{~km}^{2}$. Conforme o Instituto Brasileiro de Geografia e Estatística (IBGE) de 2020, a população de Vitória da Conquista é de 341.128 habitantes. A coleta de dados foi realizada em pontos estratégicos da cidade, onde existe um alto fluxo de pessoas que residem em diversos bairros.

Participaram 207 pessoas que foram abordadas no centro do munícipio de Vitória da Conquista de forma aleatória. Foram incluídos no estudo indivíduos maiores de 18 anos, de ambos os sexos, contando como critério de exclusão de crianças e adolescentes, pois esses possuem pouca probabilidade de serem os responsáveis pelo descarte de medicamentos de suas residências. Inicialmente, foi feita uma breve explicação do projeto e, caso o indivíduo demonstrasse interesse em participar, o Termo de Consentimento Livre e Esclarecido era devidamente apresentado, e a coleta de dados realizada.

Para a coleta de dados, foi utilizado um instrumento estruturado contendo 11 questões que abordam o perfil socioeconômico demográfico dos participantes, bem como, o grau de conhecimento acerca do descarte de medicamentos vencidos/em desuso. Foram abordadas questões como: idade, gênero, presença de medicamentos na casa do entrevistado, se verificam a validade do medicamento, forma de descarte dos medicamentos vencidos, destino correto dos medicamentos vencidos e possíveis motivos do vencimento. Para a organização e análise dos dados coletados através das abordagens em campo, foi utilizado o programa Microsoft Excel 2019, realizando uma análise através de estatística descritiva.

Em atendimento aos dispostos na Resolução 466/12, a pesquisa foi apreciada pelo Comitê de Ética e Pesquisa (CEP) da Faculdade Independente do Nordeste - CEP/FAINOR, sendo iniciada após aprovação sob parecer n. 51404221.8.0000.5578.

\section{Resultados e Discussão}

Após a coleta e análise dos dados, foi observado que a maioria dos participantes, $47 \%$ (n: 98), possuem idade entre 18 e 30 anos e, a minoria 6,7\% (n: 14), possuem 60 anos ou mais. Essa distribuição por idade pode estar relacionada ao fato da coleta de dados ter sido realizada durante a pandemia do novo coronavírus, quando ainda havia a orientação para idosos evitarem a exposição em locais públicos. Dentre os entrevistados, foi observado uma maior predominância do gênero feminino (52,17\%), essa prevalência pode ser justificada pelo fato de que 51,82\% da população de Vitória da Conquista são do sexo feminino (IBGE, 2010). Avaliou-se que $92,75 \%$ da população estudada possui medicamentos em casa afirmando ter um 
estoque domiciliar, também conhecido como farmácia caseira. Silva e Almeida (2017) também apontaram essa preeminência em um estudo realizado em Sete Lagoas-MG, onde 97,62\% relataram ter medicamentos em suas residências. Esse alto índice revela a facilidade de aquisição de medicamentos que, muitas vezes, podem ser obtidos sem a necessidade de prescrição médica.

Tabela 1: Dados sociodemográficos e conhecimento dos participantes acerca dos medicamentos e seu descarte.

\begin{tabular}{lcc}
\hline Variáveis & $\mathbf{N}$ & \% \\
\hline Idade & 98 & 47,34 \\
$18-30$ anos & 37 & 17,87 \\
$31-40$ anos & 38 & 18,36 \\
$41-50$ anos & 20 & 9,66 \\
51 a 60 anos & 14 & 6,77 \\
60 anos ou mais & & \\
Gênero & 108 & 52,17 \\
Feminino & & \\
Possui medicamento em casa? & 192 & 92,75 \\
Sim & & \\
Verificam a validade do medicamento? & 179 & 86,47 \\
Sim & & \\
Possíveis motivos do vencimento & 44 & 21,26 \\
Fim do tratamento & 128 & 61,83 \\
Longo período de armazenamento & 35 & 16,91 \\
Interrupção do uso & & \\
& &
\end{tabular}

O que faz com as sobras de medicamentos na sua residência

Devolve à unidade de saúde ou ao agente de saúde $\quad 8 \quad 3,86$

$\begin{array}{lll}\text { Guarda para usar outra vez } & 107 & 51,69\end{array}$

$\begin{array}{lll}\text { Coloca no lixo } & 75 & 36,23\end{array}$

$\begin{array}{llr}\text { Dá aos vizinhos/amigos/parentes } & 17 & 8,22\end{array}$

Como descarta os medicamentos vencidos ou em desuso?

$\begin{array}{lcc}\text { Junto ao lixo doméstico } & 136 & 65,70 \\ \text { Na pia } & 6 & 2,90 \\ \text { No vaso sanitário } & 19 & 9,18 \\ \text { Posto de saúde / Farmácia / Centro Comunitário } & 32 & 15,46 \\ \text { Nunca descartou } & 14 & 6,76\end{array}$

Quais os medicamentos descartados com maior frequência?

$\begin{array}{lcc}\text { Antibióticos } & 43 & 20,77 \\ \text { Analgésicos } & 72 & 34,78 \\ \text { Anti-inflamatórios } & 33 & 15,94 \\ \text { Antidepressivos } & 0 & 0 \\ \text { Outro } & 56 & 27,06 \\ \text { Não descarta } & 3 & 1,45\end{array}$




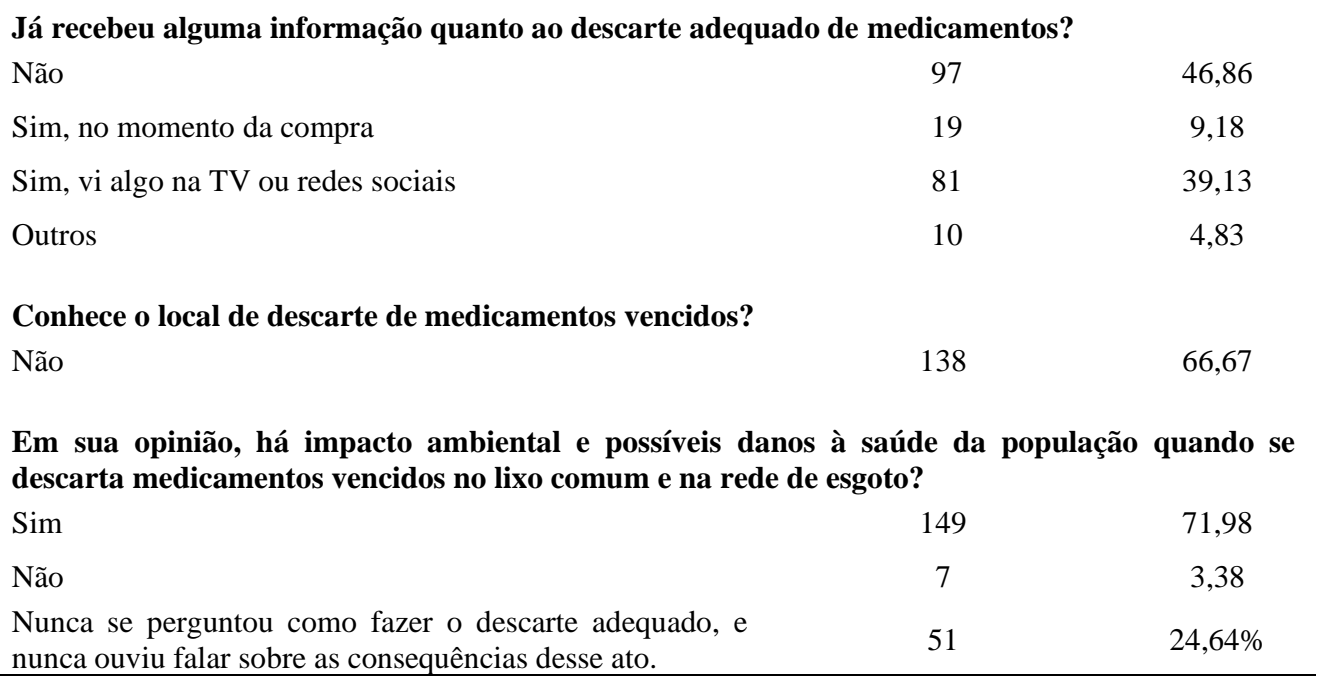

Fonte: Autores.

Quanto à presença de medicamentos em estoque domiciliar, a verificação do prazo de validade é realizada por $86,47 \%$ dos entrevistados, tendo em vista que, apenas 13,53\% relataram nunca verificar a validade. Um estudo realizado por Ramos et al (2017), em Brasília-DF comprovou esse alto índice, visto que, 75,2\% dos participantes certificam-se desse prazo. Os medicamentos administrados na própria residência, quando vencidos, trazem riscos à saúde no caso de ingestão não acidental e acidental por idosos ou crianças, podendo ser os responsáveis por possíveis intoxicações, resistências e efeitos indesejáveis.

Quando questionados sobre os possíveis motivos do vencimento, 61,83\% dos interrogados mencionaram o longo período de armazenamento, 21,26\% o fim do tratamento e 16,91\% a interrupção do uso. Com relação às sobras de medicamentos após realização do tratamento, 51,69\% disseram que os guardam para posterior reutilização, 36,23\% afirmaram colocar no lixo, 8,22\% relataram dar aos vizinhos, amigos e parentes e 3,86\% devolvem para alguma unidade ou agente de saúde. Em contrapartida, resultados próximos a estes foram encontrados por Cruz et al (2017), em estudo nos municípios do Vale do Jequitinhonha - Minas Gerais, onde 46,7\% dos entrevistados armazenaram fármacos em casa para usos futuros.

No que diz respeito ao descarte total de medicamentos vencidos ou em desuso, $65,70 \%$ disseram que desprezam no lixo comum, 15,46\% devolvem ao posto de saúde/farmácia/centro comunitário, 9,18\% descartam no vaso sanitário, 2,90\% relataram descartar na pia e 6,76\% nunca descartaram. Durante a análise dos dados verificou-se que o principal destino dos medicamentos vencidos é o descarte no meio ambiente. Kusturica et al, 2017, observou que em diversos países a população descarta os medicamentos de forma inadequada, afetando assim, o ecossistema. Pesquisa realizada em um município do oeste catarinense, por Zanatta et al (2021) mostrou resultados semelhantes ao encontrado no presente estudo onde $41 \%$ desprezam os medicamentos vencidos no lixo comum, $22 \%$ devolvem à unidade básica de saúde, $5 \%$ descartam no vaso sanitário e os demais resultados referem-se a outros destinos.

Pôde-se observar que dentre os medicamentos que os entrevistados mais descartam, destacaram-se os analgésicos com $34,78 \%$, outros (antialérgicos, anticoncepcionais, xaropes, pomadas, não especificados) com 27,06\%, antibióticos com 20,77\% e anti-inflamatórios com 15,94\%. Já a classe dos antidepressivos obtiveram um total de 0\%, onde os participantes relataram não descartá-los. Por outro lado, em um estudo realizado por Mendonça (2016), notou-se que os medicamentos que os entrevistados mais descartam são antibióticos (65\%), analgésicos (9\%), anti-inflamatórios (7\%), outros (19\%).

Atualmente, o efeito desses fármacos no meio ambiente é uma questão que gera muitos levantamentos e discussões. De acordo com a Agência Brasileira de Desenvolvimento Industrial - ABDI (2013), a contaminação do meio ambiente tem dentre suas principais causas o descarte incorreto de medicamentos, que ao serem descartados no lixo comum ou rede de 
esgoto, os medicamentos podem contaminar o solo, as águas superficiais, e também as águas subterrâneas. Essas substâncias no ambiente podem transformar-se em substâncias tóxicas, afetando o meio ambiente e contribuindo para o desenvolvimento de resistência bacteriana e, portanto, afetando a qualidade de vida e saúde da população (Zanatta et al, 2019). Conforme Carvalho et al. (2009), segundo pesquisa realizada nos Estados Unidos e publicada em 2008, cerca de 41 milhões de cidadãos americanos, em 24 áreas metropolitanas, recebem água potável contaminada por uma variedade de produtos farmacêuticos, como antibióticos e hormônios.

Quando perguntados se haviam recebido alguma informação sobre o descarte adequado de medicamentos, 46,86\% dos participantes revelaram não receber, 39,13\% disseram que sim, onde adquiriram informações por meio da TV ou redes sociais, 9,18\% relataram que receberam informações no momento da compra e 4,83\% informaram ter recebido informações na faculdade ou através de conversas com amigos. Em um estudo realizado por Silva e Almeida, 2016, 47,6\% dos entrevistados responderam já terem recebido informações quanto ao descarte de medicamentos, enquanto $52,4 \%$ disseram nunca terem recebido tais informações.

Outro questionamento foi acerca do conhecimento do local no qual é realizado o descarte de medicamentos vencidos, onde $66,67 \%$ relataram não conhecê-lo. Através dessa pergunta é possível observar que a maior parte dos entrevistados não tem conhecimento sobre os locais de descarte dos medicamentos. Isso acontece em decorrência a falta de informação sobre os malefícios causados pelos fármacos ao meio ambiente e pela carência de postos de coleta.

Por fim, os entrevistados foram questionados se acreditavam haver impacto ambiental e possíveis danos à saúde da população quando se descarta medicamentos vencidos no lixo comum e na rede de esgoto, onde $71,98 \%$ disseram haver impactos ambientais, 24,64\% nunca se perguntaram como fazer o descarte adequado, e nunca ouviram falar sobre as consequências desse ato e 3,38\% disseram não acreditar que este ato poderia gerar consequências ao meio ambiente. Esses resultados se assemelharam ao estudo realizado por Gasparini et al (2011), que verificou as seguintes opiniões dos entrevistados quanto à consciência ambiental: 80,4\% acham que essa atitude gera problemas ambientais e 84,55\% relatam que nunca receberam nenhuma informação sobre esse assunto. Esses dados apontam que a maior parte dos entrevistados tem consciência ambiental e acreditam que o descarte inadequado leva a problemas como a contaminação do solo e da água. Apesar de acreditarem nos impactos causados por essa prática, grande parte dos entrevistados também mencionaram descartar os medicamentos no lixo comum ou na rede de esgoto. Essa divergência pode ser justificada pela falta de orientação acerca da forma correta de realizar esse descarte, porém, uma vez que a população esteja instruída quanto aos impactos do descarte incorreto, as unidades de saúde devem estar preparadas para receber os medicamentos e realizarem a logística reversa, através da abertura de postos de coleta e criação de campanhas que orientem sobre o uso racional dos medicamentos (Lenhardt el al., 2014).

\section{Considerações Finais}

Os resultados encontrados no presente estudo evidenciam que a população de Vitória da Conquista descarta os medicamentos de forma incorreta. Isso nos mostra que há um déficit de conhecimento acerca do descarte adequado em decorrência da falta de informação sobre o tema, carência de pontos de coleta e falta de divulgação dos problemas ambientais provocados pelo descarte. Esses dados mostram a gravidade do cenário atual relacionado ao descarte de medicamentos nas residências e a importância de se estabelecer medidas sociais, educativas e ambientais, que ensinem à população formas corretas de descarte de medicamentos.

O descarte incorreto de medicamentos pode ser notado em vários estudos, contudo, as orientações e informações sobre as consequências desse ato ainda são insuficientes para a mudança dessa realidade. Por essa razão, tem-se a logística reversa que visa uma maior preservação ambiental. No entanto, mesmo após esse sistema ser colocado em prática os 
profissionais farmacêuticos exercem um papel imprescindível na orientação sobre o acúmulo e descarte desses resíduos, minimizando a falta de informação por grande parte da população, e consequentemente, evitando a contaminação do meio ambiente.

O presente estudo apresentou limitações importantes quanto à sua amostra. Dentre essas limitações, está o número reduzido de participantes da pesquisa, o que minimiza a capacidade dos pesquisadores de realizarem inferências sobre a população total do município. Outra limitação encontrada, foi o fato de que, a coleta ao ser realizada em apenas dois pontos estratégicos da cidade, impossibilita conhecer a característica da população de forma geral.

\section{Referências}

Agência Brasileira de Desenvolvimento Industrial (ABDI). (2013). Logística Reversa para o setor de medicamentos. Brasília: CRFMG.

Bandeira, E. O., Abreu, D. P. G., Lima, J. P., Costa, C. F. S., Costa, A. R., \& Martins, N. F. F. (2019). Medicine dispoasal: a socio-enviromental and health issue/Descarte de medicamentos: uma questão socioambiental e de saúde. Revista de Pesquisa Cuidado é Fundamental Online, 11(1), 1-10. doi:https://doi.org/10.9789/2175-5361.2019.v11i1.1-10

Carvalho, E. V., Ferreira, E., \& Santos, C. (2009). Aspectos legais e toxicológicos do descarte de medicamentos. Revista Brasileira de Toxicologia, 22, 1-8.

Constantino, V. M., Fregonesi, B. M., Tonani, K. A. A., Zagui, G. S., Toninato, A. P., Nonose, E. R. S., Fabriz, L. A., \& Segura-Muñoz, S. I. (2020). Estoque e descarte de medicamentos no domicílio: uma revisão sistemática. Ciência \& Saúde Coletiva (Ribeirão Preto), 25, 585-594. doi:https://doi.org/10.1590/141381232020252.10882018

Cruz, M. J. B., Azevedo, A. B., Cruz, H. L., Bodevan, E. C., Araujo, L. U., \& Santos, D. F. (2017). Descarte de medicamentos em municípios do Vale do Jequitinhonha, Minas Gerais, Brasil. Revista Vigilância Sanitária em Debate: Sociedade, Ciência \& Tecnologia (Diamantina), 5(1), 84-90. doi:https://doi.org/10.22239/2317-269X.00802

Paula, V. G., Tormin, C. V., \& Souza, D. S. (2019). Descarte Adequado De Medicamentos uma Responsabilidade Social Compartilhada. Educação: Saberes e Prática (Luziânia), 8(1).

Ferreira, F. C., Behenck, A. C. M., Costa, D. A., Feuser, Z. P., \& Borges, M. S. (2020). Práticas extensionistas auxiliando no descarte correto de medicamentos. Interagir: pensando a extensão (Rio de Janeiro), (29), 120-131. doi:https://doi.org/10.12957/interag.2020.52361

Gasparini, J. C., Gasparini, A. R., \& Frigieri, M. C. (2011). Estudo do descarte de medicamentos e consciência ambiental no município de Catanduva-SP. Ciência \& Tecnologia: FATEC-JB (Jaboticabal), 2(1), 38-51.

Instituto Brasileiro de Geografia e Estatística (IBGE). (2012). Censo Brasileiro de 2010. Rio de Janeiro.

Kusturica, M.P., Tomas, A., Sabo, A. Disposal of unused drugs: knowledge and behavior among people around the world. (2017). Rev Environ Contam Toxicol. 240, 71-104. https://doi.org/10.1007/398_2016_3

Lenhardt E. H., Sólis, L. J. B., Cintra, E. V. C. S., \& Botelho, E. H. L. (2014). O Descarte de Medicamentos no Bairro Grande Terceiro, Cuiabá-MT. Journal of Health Sciences, 16(1), 5-8. doi: https://doi.org/10.17921/2447-8938.2014v16n1p\%25p.

Lopes, B. A., Drombovski, L., Rosa, P. B., Sestile, C. C., Tenfen, A., \& Neis, V. B. (2021). Avaliação nos cuidados com armazenamento e descarte de medicamentos por acadêmicos de uma Faculdade do Sul do Brasil. Brazilian Journal of Development, 7(1), 7783- 7797. doi:https://doi.org/10.34117/bjdv7n1528

Marcondes, R. C., Miguel, L. A. P., Franklin, M. A., \& Perez, G. (2017). Metodologia para trabalhos práticos e aplicados. São Paulo: Editora Mackenzil.

Mendonça, J. M. (2016). Diagnóstico Acerca do Descarte de Medicamentos no Município de Nova Palmeira - PB (Trabalho de Conclusão de Curso, Universidade Estadual da Paraíba, Campina Grande - PB). http://dspace.bc.uepb.edu.br/jspui/handle/123456789/10154

Prodanov, C. C., \& De Freitas, E. C. (2013). Pesquisa Científica. Metodologia do trabalho científico: métodos e técnicas da pesquisa e do trabalho acadêmico-2 ${ }^{a}$ Edição(26-90). Novo Hamburgo, Rio Grande do Sul, Brasil: Editora Feevale.

Ramos, H. M. P., Cruvinel, V. R. N., Meiners, M. M. M. A., Queiroz, C. A., \& Galato, D. (2017). Descarte de medicamentos: uma reflexão sobre os possíveis riscos sanitários e ambientais. Ambiente \& Sociedade (São Paulo), 20(4), 145-168.

Silva, R. E., \& Almeida, A. F. S. (2017). Panorama do Descarte de Medicamentos Domiciliares no Município de Sete Lagoas/MG. Revista Brasileira de Ciências da Vida (Sete Lagoas), 5(1).

Tonet, A, G., Oliveira, A. K. M., Rivero-Wendt, C. L. G., Araújo, G. M., Brito, I. K., Ganassina, A. R.; \& Matias, R. (2020). Medicamentos vencidos ou em desuso e riscos ambientais no município de Terenos, Mato Grosso do Sul. Ensaio e ciência (Terenos), 24(24), p. 170-182. doi:https://doi.org/10.17921/14156938.2020v24n2p170-182

Zanatta, L., Bello, A. P. D., \& Carraro, D. R. (2019). Resíduos de Medicamentos e Perfurocortantes em Lixo Comum e os Riscos à Saúde dos Catadores de Materiais Recicláveis. Prevenção e promoção de saúde. Atena Editora (Ponta Grossa), 8, 102-113. doi:https://doi.org/10.22533/at.ed.4341918128

Zanatta, L., Zenatti, S. T., Korb, A., \& Cabral, D. B. (2021). Levantamento Sobre Formas de Descarte de Medicamentos Vencidos e em Desuso Pela População de mm Município do Oeste Catarinense. In Congresso Internacional em Saúde. 